\title{
Non-invasive measurements of the dry solids content of whole potatoes using unilateral magnetic resonance: towards automation
}

Elizabeth R. Dye, Theodore Hughes-Riley, Michael I. Newton and Robert H. Morris

Nottingham Trent University, School of Science and Technology, Clifton Lane, Nottingham, NG11 8NS, UK.

Corresponding Author: rob.morris@ntu.ac.uk.

Crisps or chips are considered a popular snack food around the world and at their most fundamental are potatoes which are sliced and then fried. It has been known for some time that during their production industrially, controlling the final oil content requires prior knowledge of the dry solids of the potatoes to modify the temperature and frying time to give the best product. The dry weight of a batch of potatoes is most commonly performed using a buoyancy measurement. In preliminary experiments, we have found evidence that such a measurement, whilst representative of the average dry solids, does not offer the most appropriate measurement since the variation within the batch is significant. We present an investigation into the properties of intact potatoes using magnetic resonance relaxation measurements and relate these to the dry solids content. This preliminary study will lay the groundwork for the development of an online process monitoring device based around a unilateral sensor to allow batch sorting of incoming potatoes.

\section{Introduction}

ried sliced potatoes, known as crisps or chips, are a popular food product throughout the world. To ensure that the highest quality product is produced, before frying, the dry solids content of the potato must be known. This allows for changes to the temperature and make-up of the frying oil. Within the industry, such a measurement is typically made by bulk buoyancy $^{1}$ measurements of large batches even though the variation within a typical batch is significant.

Work by Thybo et al. ${ }^{2}$ has investigated various magnetic resonance (MR) parameters for the purposes of identifying dry matter content in potatoes seeing strong correlations with the spin-lattice relaxation time $\left(T_{1}\right)$ and the weighting of the second component of the bi-exponential spin-spin relaxation time $\left(\mathrm{T}_{2}{ }^{\text {eff }}\right)$ fitting. Chen et al. ${ }^{3}$, in their review of the work however state that the correlation was not satisfactory. Further investigation by Thybo et al. ${ }^{4}$ has shown promising results regarding $T_{2}{ }^{\text {eff }}$ values (the time constant acquired from a Carr-Purcell-Meiboom-Gill (CPMG) sequence experiment $\mathrm{t}^{5}$ ) using a commercial low-field nuclear magnetic resonance (NMR) system, although this system was only able to scan a small sample (diameter $11.5 \mathrm{~mm}$ ) which was removed from the potato. Building a large, low-cost magnetic resonance system is possible ${ }^{6}$, although these systems generally have large field gradients which lead to significant shortening of the $T_{2}^{\text {eff }}$ parameter. It is possible to reduce these gradients through magnet optimisation but this is a costly process.

In this work, the relationship between dry solid content and magnetic resonance relaxation parameters in raw potatoes is explored further using a commercial unilateral instrument (Profile NMRMOUSE $^{\circledR}$, ACT Aachen, Germany) ${ }^{7}$. The Profile NMR-MOUSE ${ }^{\circledR}$ has previously been used to investigate food systems in the literature including investigation of food emulsions ${ }^{8,9}$ and olive oil adulteration $^{10}$. This work lays the groundwork for the development of a low-cost online monitoring system to be used as an alternative to the traditional approaches to determining the dry solid content.

\section{Methodology \\ Sample preparation}

Maris Piper potatoes (wet weights of between 100g and 300g) were used in this study as they were readily available and had similar attributes to those used for crisp production ${ }^{11}$. In order to determine the dry solids content of the samples, whole potatoes were weighed using an electronic balance (Kern \& Sohn GmbH, Balingen, Germany) before being sliced into four segments and placed in a convection oven (FED 53, Binder GmbH, Tuttlingen, Germany) at a temperature of $100^{\circ} \mathrm{C}$. This temperature was used to ensure the quickest removal of water without cooking the potatoes and changing their internal structures. The potato quarters were left to dry for no less than 15 hours to ensure that all of the moisture had been removed (a time series of mass vs dry time had been previously conducted, with a maximum plateau time of 10 hours). Following this, the dry masses of the slices were recorded and compared to the wet mass to determine the fraction of dry solids.

\section{Magnetic resonance}

Magnetic resonance (MR) experiments were conducted using a Profile NMR-MOUSE ${ }^{\circledR}$ paired with a NMR spectrometer (Apolo LF, Tecmag, Houston, TX, USA). The Profile NMR-MOUSE ${ }^{\circledR}$ employs a unilateral permanent magnet geometry which generates a thin slice $(\sim 100 \mu \mathrm{m})$ of uniform magnetic field at 0.25T a configurable distance from the surface. Large samples, significantly greater than the dimensions of the unit are placed atop the magnet. The thin selected volume then resides some distance into the surface of the sample. The Profile NMR-MOUSE ${ }^{\circledR}$ used in these experiments has a gradient of $11.38 \mathrm{~T} / \mathrm{m}$ and was set for its deepest distance into the sample, approximately $5 \mathrm{~mm}$ from the surface. In this work, we 


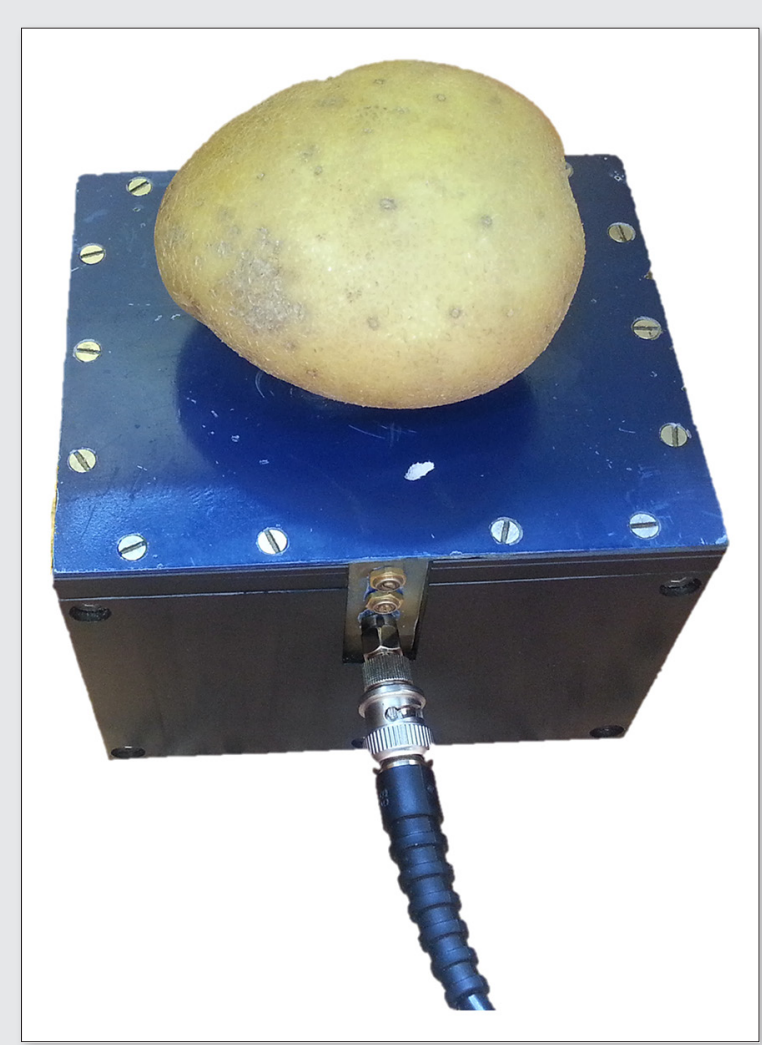

Figure 1. A photograph of the Profile NMR-MOUSE ${ }^{\circledR}$ with a potato placed on top of it. This was the experimental set-up for the magnetic resonance experiments conducted within this study.

placed whole potatoes on the surface of the instrument for measurements (see Fig. 1).

The inhomogeneity of the field surrounding the selected slice leads to significant off resonance excitation rendering many traditional magnetic resonance pulse sequences prone to significant artefact. Although capable of imaging, in this work, we used the system to make relaxation measurements of both $T_{1}$ and $T_{2}{ }^{\text {eff }}$. The strong field gradient of the system makes it ideal for measurements of the self-diffusion coefficient of the system's protons. Although not shown here, a series of measurements of diffusion were made on potatoes but little variation was found between samples.

The CMPG sequence ${ }^{4}$ is frequently used on such instruments as it allows collection of useful signals even in the presence of significant inhomogeneity. In this work, we use this sequence to acquire both of the relaxation parameters of interest, $T_{1}$ and $T_{2}$ eff , of the raw potatoes. $T_{2}^{\text {eff }}$ measurements were made using the parameters in Table 1. The value of the $T_{2}$ eff parameter were determined by fitting a multi-exponential to the integrals of the echoes. $\tau_{E}=100 \mu \mathrm{s}, 1024$

Table 1. Experimental Parameters used for unilateral magnetic resonance measurements

\begin{tabular}{lcc}
\hline Parameter & Value $\mathrm{T}_{2}^{\text {eff }}$ & Value $\mathrm{T}_{1}$ \\
\hline Echo Time & $100 \mu \mathrm{s}$ & $100 \mu \mathrm{s}$ \\
Number of Echoes & 1024 & 32 \\
\hline Repetition Time(s) & $1200 \mathrm{~ms}$ & $50 \mathrm{~ms}$ to 3s in \\
$200 \mathrm{~ms}$ steps \\
Averages & 64 & 16
\end{tabular}

echoes, and 64 experimental averages, with $\mathrm{T}_{2}{ }^{\text {eff }}$ values extracted by exponentially fitting the echo peaks. For $T_{1}$ measurements, the repetition time (the time between $90^{\circ}$ pulses) was varied to perform a saturation recovery ${ }^{12}$ with the parameters in Table 1. Multiple echoes were summed after integration to improve the signal-tonoise ratio (SNR). This is only valid where minimal decay is seen in the echo train to prevent $T_{2}$ weighting of the result, hence in these experiments, the first 32 echoes only are summed for the minimum echo time of which the system is capable. The integral of the echo train is plotted against the repetition time and a multi-exponential fit used to determine the $T_{1}$ relaxation parameter.

In all experiments, the exponential fitting and graph production was performed in Matlab (The MathWorks Inc., Natick, MA, USA). The need for rapid collection of data and the low SNR of the chosen MR hardware limits the reliability of inverse-laplace type techniques for analysis.

\section{Results and discussion}

Potatoes were taken from a single batch with an average dry solids content around $20 \%$. As can be seen in the measurements presented, this provided us with samples ranging between $18 \%$ and $21 \%$. No correlation was found between the dry content and the initial weight of the potatoes which is to be expected.

The $\mathrm{T}_{2}^{\text {eff }}$ data of the raw potatoes in Fig. 2 shows a relationship between the dry solid fraction and the relaxation parameter, with increasing fraction leading to a shorter value. This corresponds to a reduction in the mean free path leading to a shortening of the $T_{2}$ time. Unfortunately, the strong gradient present on the unilateral system employed in this work leads to a very short value of the $T_{2}{ }^{\text {eff }}$ parameter. This makes it very challenging to successfully find multiple decay components as have been found in the literature ${ }^{2,4}$. In future studies, we will endeavour to reduce this gradient to increase the value of $\mathrm{T}_{2}$ eff to the point at which an inversion technique can be successfully employed to determine the individual relaxation components.

Fig. 3 shows a stronger relationship between $T_{1}$ of a raw potato and its dry solid content, with $T_{1}$ varying between $1005 \mathrm{~ms}$ and $683 \mathrm{~ms}$ for dry solids fractions of 0.181 and 0.207 respectively. While the trend observed for the 0.181 to 0.205 range of dry solids fractions is somewhat linear, higher dry solid fractions see a more rapid shortening of the $T_{1}$ time. Previous work ${ }^{2}$ showed a correlation

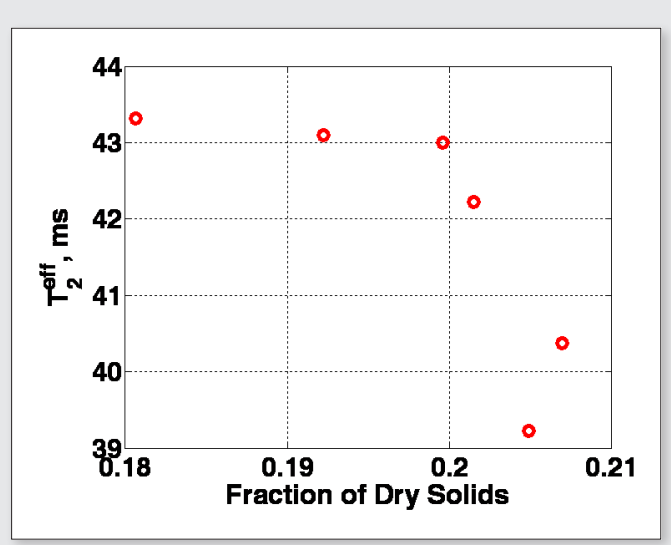

Figure 2. Raw potato $T_{2}^{\text {eff }}$ time as a function of the fraction of dry solids. As with the $T_{1}$ times, $T_{2}^{2}$ eft times were seen to reduce as a function of the fraction of dry solids, with small changes in relatively high fractions of $d r y$ solids resulting in the largest changes in the $\mathrm{T}_{2}{ }^{\text {eff }}$ time. 


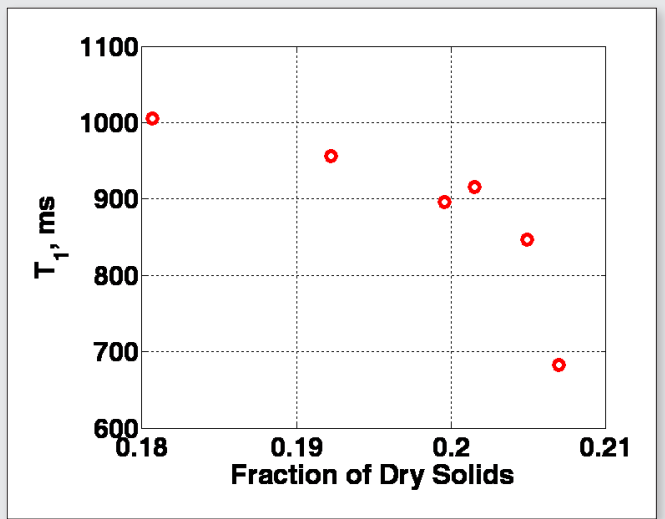

Figure 3. Raw potato $T_{1}$ relaxation time as a function of the fraction of dry solids. $T_{1}$ times were seen to reduce as a function of the fraction of dry solids, with small changes in relatively high fractions of dry solids resulting in the largest changes in the $T_{1}$ relaxation time.

between dry solids fraction and $T_{1}$ time with a regressor of -0.85 . In this work, we found a non-linear relationship which cannot therefore be directly compared with previous measurements. One contributing factor in the reduction of $T_{1}$ with an increase in dry mass fraction will be that the pore size within the potatoes will decrease as the dry mass fraction increases. The $T_{1}$ of water in porous media is known to be pore size dependant making it a useful tool in rock porometery ${ }^{13}$. In this situation however the potato starch also contains hydrogen, and the starch content will also depend on the dry mass content, making a direct pore size estimate based on $T_{1}$ problematic. Although this was expected to lead to a two component exponential decay, the fitting performed in this work suggested a monoexponential decay. In further work, we will investigate different repetition time sampling strategies to see if there is a significantly shorter $\mathrm{T}_{1}$ component which represents the starch bound water.

\section{Conclusion}

We have demonstrated useful relationships between the dry solids content of Maris Piper potatoes and two relaxation parameters, $T_{1}$ and $T_{2}$ eff. In particular, the $T_{1}$ parameter provides a good variation over the dry solids measured which increases the ease of future automation. As $T_{1}$ measurements are not particularly sensitive to large magnetic field gradients it is probable that a suitable low-cost unilateral MR sensor could be produced for this application without the need to optimise the field gradients. This technique shows promise as an alternative to the traditional methods for determining the dry solid content for the production of crisps or chips and should allow for an automated sorting process to be employed improving the quality of the end product. The location of the sensitive MR volume was likely to be within the cortex structure (the outer layers) of the potato. Although the measurements which have presented in this work show a relationship which could be exploited for automatic sorting of potatoes by dry weight, further investigation into the variation within the different structures of the potato and indeed for different cultivars is an important next step. Furthermore, confirmation that the self-diffusion coefficient does not vary significantly with dry solids should also be undertaken within the medulla structures (the central areas of the potato) as these are less coherent and likely to present with a greater variation of diffusion length as a function of solids content.
The variation which we have found within even small batches of potatoes, suggests that optimum processing is only achievable if batches of potatoes can be sorted by their dry solids content to be delivered to different fryers appropriate for the moisture level of the potatoes.

\section{Acknowledgements}

The authors would like to thank both the Ogden Trust and the East Midlands Branch of the Institute of Physics for funding the expenses necessary to present this work at the MR Food conference in Karlsruhe.

\section{References}

1. M. Nissen, "The weight of potatoes in water." American Potato Journal 32.9, 332-339 (1955).

2. A.K. Thybo, H.J. Andersen, A.H. Karlsson, S. Dønstrup, and H. Stødkilde-Jørgensen, "Low-field NMR relaxation and NMR-imaging as tools in differentiation between potato sample and determination of dry matter content in potatoes", LWT-Food Science and Technology, 36(3), 315322 (2003). doi: http://dx.doi.org/10.1016/S0023-6438(02)00210-4

3. Q. Chen, C. Zhang, J. Zhao,and Q. Ouyang, "Recent advances in emerging imaging techniques for non-destructive detection of food quality and safety", TrAC Trends in Analytical Chemistry, 52, 261-274 (2013). doi: http://dx.doi.org/ 10.1016/j.trac.2013.09.007

4. C.L. Hansen, A.K. Thybo, H.C. Bertram, N. Viereck, F. van den Berg, and S.B. Engelsen, "Determination of dry matter content in potato tubers by low-field nuclear magnetic resonance (LF-NMR)", Journal of Agricultural and Food Chemistry, 58(19), 10300-10304 (2010). doi: http://dx.doi. org/10.1021/jf101319q

5. S. Meiboom D. Gill, "Modified Spin-Echo Method for Measuring Nuclear Relaxation Times", Review of Scientific Instruments, 29, 688-691 (1958).

6. B.P. Hills, K.M. Wright and D.G. Gillies. "A low-field, low-cost Halbach magnet array for open-access NMR." Journal of Magnetic Resonance, 175(2), 336-339 (2005). doi: http://dx.doi.org/10.1016/j. imr.2005.04.015

7. G. Eidmann, R. Savelsberg, P. Blümler and B. Blümich. "The NMR MOUSE, a mobile universal surface explorer." Journal of Magnetic Resonance Series A, 122(1), 104-109 (1996). doi: http://dx.doi.org/10.1006/ imra.1996.0185

8. H.T. Pedersen, S. Ablett, D.R. Martin, M.J.D. Mallett and S.B. Engelsen. "Application of the NMR-MOUSE to food emulsions" Journal of Magnetic Resonance, 165(1), 49-58 (2003). doi: http://dx.doi.org/10.1016/ S1090-7807(03)00243-X

9. A.M. Haiduc, E.E. Trezza, D. van Dusschoten, A.A. Reszka, and J.P.M. van Duynhoven. 'Non-invasive 'through-package' assessment of the microstructural quality of a model food emulsion by the NMR MOUSE." LWT-Food Science and Technology, 40(4), 737-743 (2007). doi: http://dx.doi.org/10.1016/j.lwt.2006.02.026

10. X. Zheng, R.H. Morris, M. Bencsik, and M.I. Newton. "Detection of virgin olive oil adulteration using low field unilateral NMR", Sensors, 14(2), 2028-2035 (2014). doi: http://dx.doi.org/10.3390/s140202028

11. Varieties.ahdb.org.uk. (2016). Organisations. [online] Available at: http:// varieties.ahdb.org.uk/organisations/view/Pepsico [Accessed 23 Aug. 2016].

12. E. Fukushima, and S.B. Roeder, Experimental pulse NMR: a nuts and bolts approach. Addison-Wesley Reading, (1981).

13. G.H. Sørland, K. Djurhuus, H.C. Widerøe, J.R. Lien, and A, Skauge. "Absolute pore size distributions from NMR." Diffusion Fundamentals, 5, (2007): 4-1 (2007). 